\title{
A cardioplegia oxigenada na proteção miocárdica durante a cirurgia cardíaca: estudo clínico e enzimático
}

Potiguara S. da COSTA*, Sérgio Nunes PEREIRA*, Luiz B. MORAES*, Renato S. MARQUES*, Manoel A. P. ALVAREZ*, Carlos A. S. DAUDT*, Luciane M. DEBONI*, Cleonir RASKOSKI*, Mauro F. SILVA*

RBCCV 44205-69

COSTA, P. S.; PEREIRA, S. N.; MORAES, L. B.; MARQUES, R. S.; ALVAREZ, M. A. P.: DAUDT, C A. S.; DEBONI, L. M.; RASKOSKI, C.: SILVA, M. F. - A cardioplegia oxigenada na proteçâo miocárdica durante a cirurgia cardiaca: estudo clínico e enzimático. Rev. Bras. Cir. Cardiovasc. 3(3): 210-215, 1988.

RESUMO: A cardioplegia tem sido reconhecida como um fator muito importante na proteção do miocárdio. Sabe-se que, mesmo a $15^{\circ} \mathrm{C}$, o coração consome oxigênio. Testes in vitro demonstraram que a cardioplegia cristalóide libera mais oxigênio que a sangüinea. Neste estudo, foram analisadas as variaçōes hemodinâmicas, eletrocardiográficas e enzimáticas em 26 pacientes divididos em 2 grupos, nos quais a cardiplegia cristalóide de Gomes foi empregada. Grupo I: 12 pacientes (solução năo oxigenada): Grupo II: 14 pacientes (solução oxigenada). A avaliação dos pacientes incluiu a recuperação hemodinâmica após a parada cardiaca, o uso de drogas vasoativas, o ritmo e o aspecto do eletrocardiograma (ECG), a freqüência cardiaca (FC), a pressão arterial média (PAM), a pressão venosa central (PVC) e as enzimas TGO e CPK-MB. Estes parâmetros foram medidos nos seguintes tempos: antes, logo após a cirurgia e após 6 . 12. 24,48 e 72 horas de pós-operatório. Os resultados demonstraram que a recuperaçāo hemodinâmica foi similar em ambos os grupos. O uso de drogas vasoativas foi maior no Grupo II. No ECG, observou-se mais bradicardia e fibrilação ventricular no Grupo II do que no Grupo I. A frequeência cardiaca, a pressão arterial média e a pressão venosa central não mostraram diferença significativa em ambos os grupos. As enzimas TGD e CPK-MB mostraram elevação mais acentuada no Grupo I do que no II e essa diferença foi significativa $(P<0,01)$. Em conclusão, os dados sugerem que houve comportamento semelhante em ambas as soluçôes, quanto à recuperação hemodinâmica e a parâmetros vitais. Observou-se uma incidência maior de bradicardia no Grupo II, provavelmente relacionada à maior duração da parada cardiaca, com maior número de infusōes de solução cardioplégica. A variaçāo das enzimas sugere que a solução oxigenada foi mais efetiva do que a não oxigenada, para a preservaçâo do miocárdio.

DESCRITORES: proteção miocárdica, cardioplegia.

\section{INTRODUÇĀO}

A importância da solução cardioplégica em cirurgia cardiaca é reconhecida por todas as equipes que, atualmente, procuram aperfeiçoar a sua composição pela adiçảo de componentes com vistas à obtenção de melhores resultados.
Um dos aspectos mais estudados diz respeito ao uso de soluçōes oxigenadas, pois se sabe que, mesmo parado, o coraçāo consome oxigênio ${ }^{3,9}$. FOLLETTE et alii $^{3}$ defenderam o emprego de cardioplegia com sangue, para poder oferecer o oxigênio ao miocárdio.

Entretanto, DIGERNESS et alii ${ }^{2}$ demonstraram, in

Trabalho realizado no Hospital Universitário da Universidade Federal de Santa Maria. Santa Maria, RS, Brasil.

Recebido para publicaçăo em 1: de dezembro de 1988.

- Do Hospital Universitário da Universidade Federal de Santa Maria

Endereço para separatas: Potiguara S. da Costa. Rua Astrogildo de Azevedo, 185. 97015 Santa Maria, RS, Brasil. 
vitro, que a liberação de oxigênio era maior na solução cristalóide do que na que continha sangue e atribuiu esta diferença ao desvio da curva de dissociação da hemoglobina pelo frio, que levava a maior afinidade desta pelo oxigênio, dificultando sua liberação.

KANTER et alii ${ }^{6}$, usando um substituto de sangue (fluorocarbono), observaram maior liberação de oxigênio com esta substância do que com sangue ou solução cristalóide.

TABAYASHI et alii ${ }^{10}$ demonstraram que as soluções cristalóides, ou de fluorocarbono, oxigenadas foram superiores. à não oxigenada, após 2 horas de parada cardiaca.

LEDINGHAM et alii ${ }^{7}$, em estudo experimental, demonstraram que a oxigenação da solução cardioplégica do Hospital St. Thomas melhorou a sua capacidade de proteger o miocárdio após longos periodos de isquemia. Observaram que havia melhor estabilidade elétrica, recuperação hemodinâmica e redução da liberação da enzima CPK-MB. DE WITT et alii ${ }^{1}$ demonstraram melhora de função mecânica, captação de oxigênio e da fosforilação oxidativa, quando empregaram niveis mais elevados de oxigênio na solução cardioplégica.

Em nosso país, GOMES ${ }^{4}$ demonstrou, em estudo experimental, aumento de captaçāo de oxigênio pelo miocárdio, referindo que as soluçōes cristalóides somente liberavam oxigênio quando a pO2 estava acima de $200 \mathrm{~mm} \mathrm{Hg}$.

Mais recentemente, MURAD et alii ${ }^{8}$, analisando a proteção miocárdica sob o aspecto ultra-estrutural, verificaram que a cardioplegia com sangue foi mais eficaz do que a solução cristalóide e atribuiu a diferença à capacidade das hemácias de transportar oxigênio. Observou, ainda, que a diferença foi mais acentuada na fase de reperfusão.

Com base nos estudos acima, nos propusemos a desenvolver pesquisa avaliando as variações hemodinâmicas, eletrocardiográficas e a resposta enzimática, em pacientes submetidos a cirurgia cardíaca com circulação extracorpórea.

\section{CASUÍSTICA E MÉTODOS}

Foram estudados 26 pacientes, divididos em 2 grupos: Grupo I (controle - solução nāo oxigenada): 12 pacientes; Grupo II (soluçāo oxigenada): 14 pacientes.

\section{Técnica de Oxigenação da Solução Cardioplégica}

Ao ser preparado o aparelho coraçāo-pulmão artificial para a perfusāo, foi montado, também, um circuito para a oxigenação cardioplégica para a infusão na raiz da aorta. A solução cardioplégica empregada foi a descrita por GOMES ${ }^{4}$, que ficava circulando gelada em circuito fechado. No momento do pinçamento da aorta, era iniciada a infusão da solução a $4^{\circ} \mathrm{C}$, procurando-se manter a pressāo ao redor de $80 \mathrm{~mm} \mathrm{Hg}$. A quantidade de solução infundida foi de $300 \mathrm{ml} / \mathrm{m}^{2}$ de superfície corpórea na primeira dose e de $150 \mathrm{ml} / \mathrm{m}^{2}$ nas subseqüentes, com intervalo de 30 minutos.

\section{Coleta de Dados}

A coleta de dados foi feita por meio de um protocolo onde foram anotadas as informações referentes ao paciente, à lesão cardíaca, ao grau funcional, ao tipo de cirurgia realizada, aos tempos de perfusão e de parada cardiaca, à recuperaçāo pós-cardioplegia, às variaçōes do eletrocardiograma e ao uso de drogas vasoativas.

Foram também avaliados os seguintes parâmetros: freqüência cardiaca (FC), pressão arterial média (PAM), pressão venosa central (PVC) e os valores das enzimas creatinofosfoquinase, fração $\mathrm{MB}$ (CPK-MB) e transaminase glutâmico-oxalacética (TGO) nos seguintes tempos: 1) Pré-operatório; 2) pós-operatório imediato; 3) 6 horas de pós-operatório, 12 horas de pós-operatório, 24 horas de pós-operatório, 48 horas de pós-operatório e 72 horas de pós-operatório.

\section{Padronização dos Dados do Protocolo}

Os dados do protocolo foram padronizados de acordo com os seguintes critérios:

\section{Recuperação}

1.1 - Normal: condiçōes hemodinâmicas iguais ou meIhores que as anteriores, sem uso de drogas inotrópicas. 1.2 - Síndrome de baixo débito: presença de hipotensão moderada (PAM ao redor de $60 \mathrm{~mm} \mathrm{Hg}$ ), elevação da pressão venosa central (PVC acima de $18 \mathrm{~cm}$ de água em paciente sem doença mitral prévia ou acima de 20 $\mathrm{cm}$ de água, nos mitrais) e oligúria (diurese horária inferior a $1 \mathrm{ml} / \mathrm{kg}$ de peso).

1.3 - Choque: severa deficiência de perfusão tecidual com hipotensão acentuada, acompanhada de elevação mais acentuada da pressão venosa central e oligúria ou anúria.

\section{Drogas}

Considerou-se o uso das drogas normalmente empregadas em cirurgia cardiaca, observando-se, especialmente, o uso de substâncias vaso-ativas.

\section{Eletrocardiograma}

3.1 - Ritmo: foi analisado o ritmo logo após a cardio- 
COSTA, P. S.; PEREIRA, S. N.; MORAES, L. B.; MARQUES, R. S.; ALVAREZ, M. A. P.; DAUDT, C. A. S.; DEBONI, L M.; RASKOSKI, C. SILVA, M. F. - A cardioplegia oxigenada na proteção miocárdica durante a cirurgia cardiaca: estudo clínico e enzimático. Rev. Bras. Cir. Cardiovasc., 3(3):210-215, 1988

plegia: se sinusal, bradicardia sinusal, graus de bloqueio AV, presença de fibrilação ventricular, necessidade de desfibrilação elétrica (número e intensidade dos choques).

3.2 - Aspecto: observou-se a presença de alteraçōes da repolarização ventricular como infradesnivelamento do segmento ST, corrente de lesão e sinais de infarto.

\section{Técnicas Laboratoriais}

A determinaçāo da enzima TGO foi feita pela técnica de Reitman e Frankel e a CPK-MB pelo teste ultravioleta com inibiçāo imunológica (Merck test).

\section{Estudo Estatístico}

Os dados referentes à identificaçāo dos pacientes, tipo de lesão cardiaca, grau funcional, tipo de cirurgia e os referentes à recuperação hemodinâmica, uso de drogas e o ECG foram estudados por meio de tabelas de distribuição. Os parâmetros referentes aos tempos de perfusāo e de parada cardiaca, dados hemodinâmicos (FC, PAM e PVC) e valores enzimáticos foram analisados pelos seus valores médios e erro padrão e a variação entre as médias foi comparada pelo teste $\mathrm{T}$ de Student.

\section{RESULTADOS}

Como se pode observar na Tabela 1 , não houve variaçāo significativa entre os dois grupos, no que diz respeito à idade. A distribuição quanto ao diagnóstico, apresentada na Tabela 2, demonstrou que ambos os grupos foram semelhantes, com predomínio de insuficiência coronária e estenose mitral.

A distribuição quanto ao grau funcional também foi semelhante em ambos os grupos, como se pode observar na Tabela 3. Na Tabela 4, observou-se que o tempo de perfusão, no Grupo I, variou entre 32 e 75 minutos, com um valor médio de 52,67 minutos e, no Grupo II, o tempo variou entre 34 e 88 minutos, com média de 67,07 minutos. Houve diferença significativa entre os dois grupos $(P<0,05)$. O tempo de parada cardiaca variou entre 20 e 55 minutos no Grupo I, com média

TABELA 1

DISTRIBUICYAOO DOS PACIENTES DE AMBOS OS GRUPOS DE ACORDO COM A IDADE

\begin{tabular}{lcc}
\hline IDADE & GRUPOI(NÄOOXIG.) & GRUPO /I (OXIG.) \\
\hline Mínima & 10 & 10 \\
Máxima & 55 & 65 \\
Média & 35,25 & 46,44 \\
E.P. & 5 & 6 \\
\hline
\end{tabular}

TABELA 2 DISTRIBUICAAOO DE ACORDO COM O DIAGNÓSTICO

\begin{tabular}{lcccc}
\hline DIAGNÓSTICO & \multicolumn{2}{c}{ GRUPO I } & \multicolumn{2}{c}{ GRUPO II } \\
\hline & No & $\%$ & $\mathrm{~N}:$ & $\%$ \\
C I A & 2 & 16,6 & 2 & 15,4 \\
I Ao & 1 & 8,3 & 1 & 7,7 \\
I Co & 3 & 25,0 & 4 & 30,7 \\
E Ao & - & - & 1 & 7,7 \\
E M & 3 & 25,0 & 4 & 30,7 \\
T. Fallot & 1 & 8,3 & 1 & 7,7 \\
E P I & 1 & 8,3 & - & - \\
C I V & 1 & 8,3 & - & - \\
Disf. Pr. & 1 & 8,3 & - & - \\
\hline
\end{tabular}

$\mathrm{ClA}=$ comunicação interauricular; $\mid \mathrm{Ao}$ = insuficiència aórtica: $I C o=$ insuficiência coronária; $E A O=$ estenose aórtica; $E M$ $=$ estenose mitral; $T$. Fallot $=$ tetralogia de Fallot

TABELA 3

DISTRIBUICAOO DE ACORDO COMO GRAU FUNCIONAL

\begin{tabular}{ccccc}
\hline GRAU FUNCIONAL & \multicolumn{2}{c}{ GRUPO I } & \multicolumn{2}{c}{ GRUPO II } \\
\hline & N: & $\%$ & $\mathrm{~N}$ & $\%$ \\
II & 7 & 58,3 & 7 & 50,0 \\
III & 5 & 41,7 & 6 & 42,9 \\
IV & - & - & 1 & 7.1 \\
\hline
\end{tabular}

TABELA 4

VALORES MEDIOS DOS TEMPOS DE PERFUSÄO EDE PARADA CARDIACA EM AMBOS OS GRUPOS

\begin{tabular}{lllll}
\hline PARAMETROS & \multicolumn{2}{c}{ GRUPO I } & \multicolumn{2}{c}{ GRUPO II } \\
\hline T. Perf. & 52,67 & $(3,9)$ & 67,07 & $(4,2)^{\circ}$ \\
T. P. Card. & 33,83 & $(3,2)$ & 43,86 & $(4,2)$
\end{tabular}

$\cdot P<0,05$

de 33,83 minutos e variou entre 14 e 70 minutos no Grupo II, com média de 43,86 minutos. Não houve diferença estatisticamente significativa entre os grupos.

Os dados referentes à recuperação hemodinâmica, ao uso de drogas, às alteraçōes do ECG e à necessidade de desfibrilação foram apresentados na Tabela 5. Nesta, observou-se que, em ambos os grupos, houve recuperação normal, com apenas um caso de síndrome de baixo débito em paciente do Grupo II. No que diz respeito ao uso de drogas, observou-se que houve maior incidência no Grupo II, embora sem diferença significativa.

No ritmo do ECG, observou-se que, no Grupo II, houve maior incidência de bradicardia sinusal após a desfibrilação. O aspecto do ECG após a cardioplegia foi normal, na maioria dos casos, com apenas 2 casos de infradesnivelamento de ST no Grupo I e 1 caso, decor- 
COSTA, P. S.; PEREIRA, S. N.; MORAES, L. B.; MARQUES, R. S.; ALVAREZ, M. A. P.; DAUDT, C. A. S.; DEBONI, L M.; RASKOSKI, C.; SILVA, M. F. - A cardioplegia oxigenada na proteçāo miocárdica durante a cirurgia cardiaca: estudo clínico e enzimático. Rev. Bras. Cir. Cardiovasc., 3(3): 210-215, 1988.

TABELA 5

DISTRIBUICYAOO DE AMBOS OS GRUPOS DE ACORDO COM A RECUPERAÇĀO HEMODINAMICA, USO DE DROGAS VASOATIVAS, ECG (RITMO E ASPECTO) E NECESSIDADE DE DESFRIBILACÃO

\begin{tabular}{lrrrr}
\hline \multicolumn{1}{c}{ PARAMETRO } & GRUPO I & \multicolumn{3}{c}{ GRUPO / } \\
\hline Recuperação & No & $\%$ & N. & $\%$ \\
Hemodinâmica & 12 & 100 & 13 & 93 \\
Normal & 0 & 0 & 1 & 7 \\
S.B.D. & 12 & 100 & 14 & 100 \\
Total & & & & \\
Drogas & 3 & 25 & 3 & 21 \\
Cálcio & 1 & 8 & 0 & 0 \\
Dopamina & 0 & 0 & 3 & 21 \\
N.P.S. & 0 & 0 & 2 & 14 \\
Outras & 0 & 0 & 4 & 29 \\
Ca + Dopamina & 0 & 0 & 1 & 7 \\
Ca + N.P.S. & 12 & 100 & 14 & 100 \\
Total & & & & \\
E.C.G. & 4 & 33 & 2 & 14 \\
Ritmo sinusal & 7 & 58 & 6 & 43 \\
F.V. + Sin. & 1 & 8 & 4 & 29 \\
F.V.B. Sin. & 12 & 100 & 14 & 100 \\
Total & & &
\end{tabular}

S.B.D. = síndrome de baixo débito; N.P.S. = nitroprussiato de sódio; $\mathrm{Ca}=$ cálcio; $\mathrm{B}$. Sin. = bradicardia sinusal; F. V. $=$ fibrilação ventricular.

rente de lesão, no Grupo II. A desfibrilação foi necessária em 7 casos do Grupo I e em 10 casos do Grupo II.

A variação dos valores médios da freqüência cardíaca, arterial média e pressão venosa central foi semeIhante, nos dois grupos, sem diferença significativa ( $\mathrm{Ta}$ bela 6$)$.

A Tabela 7 demonstra a variação dos valores médios das enzimas TGO e CPK-MB. Observou-se diferença significativa $(P<0,01)$ na amostra de pós-operatório imediato tanto para TGO como CPK-MB e também na

TABELA 6

DISTRIBUIÇÃO DOS VALORES MÉDIOS DE FREQÜÊNCIA CARDIACA, PRESSĀO ARTERIAL MÉDIA E PRESSĀO VENOSA CENTRAL NOS DIFERENTES TEMPOS, EM AMBOS OS GRUPOS

\begin{tabular}{lrrrrrrr}
\hline \multicolumn{2}{c}{ PARAMETROS } & \multicolumn{2}{c}{$F C$} & \multicolumn{2}{c}{ PAM } & \multicolumn{2}{c}{ PVC } \\
\hline Grupos & \multicolumn{1}{c}{ I } & \multicolumn{1}{c}{$\|$} & I & II & I & \multicolumn{1}{c}{ I } \\
Pré & 77,3 & 83,7 & 79,1 & 91,1 & 9,6 & 9,2 \\
Pós & 115,8 & 104,9 & 84,6 & 85,4 & 9,5 & 9,6 \\
$6 \mathrm{~h}$ & 97,1 & 96,9 & 74,1 & 85,4 & 10,4 & 9,8 \\
$12 \mathrm{~h}$ & 90,9 & 96,4 & 78,3 & 77,3 & 11,4 & 10,8 \\
$24 \mathrm{~h}$ & 90,7 & 91,7 & 90,3 & 80,0 & 12,4 & 13,4 \\
$48 \mathrm{~h}$ & 97,3 & 98,2 & 93,1 & 89,2 & 11,3 & 13,9 \\
$72 \mathrm{~h}$ & 92,0 & 93,7 & 10,7 & 93,4 & 10,7 & 12,3 \\
\hline
\end{tabular}

$\mathrm{FC}=$ freqüência cardíaca; PAM = pressão arterial média; PVC $=$ pressão venosa central.
TABELA 7

DISTRIBUICAAOO DOS VALORES MÉDIOS DAS ENZIMAS TRANSAMINASE GLUTAMICO-OXALACÉTICA E CREATINO-FOSFOQUINASE, FRAÇAOO MB (CPK-MB) NOS DIFERENTES TEMPOS, EM AMBOS OS GRUPOS.

\begin{tabular}{lcccc}
\hline & \multicolumn{2}{c}{ ENZIMA } & \multicolumn{2}{c}{ CPK-MB } \\
\hline GRUPO & \multicolumn{1}{c}{ ' } & II & ' & "I \\
Pré & 15,2 & 13,8 & 6,9 & 5,0 \\
Pós & $46,0^{*}$ & 35,9 & $27,2^{*}$ & 15,6 \\
$6 \mathrm{~h}$ & 49,6 & 40,5 & $25,3^{*} \cdot$ & 17,6 \\
$12 \mathrm{~h}$ & 47,8 & 45,7 & 21,8 & 19,8 \\
$24 \mathrm{~h}$ & 46,8 & 39,9 & 18,0 & 21,7 \\
$48 \mathrm{~h}$ & 36,9 & 32,0 & 13,7 & 13,2 \\
$72 \mathrm{~h}$ & 24,1 & 28,1 & 12,2 & 8,5 \\
\hline
\end{tabular}

$\cdot \mathrm{P}<0,01$

$* P<0,05$

TGO $=$ transaminase glutâmico-oxalacética.

amostra de 6 horas de pós-operatório, $(P<0,05)$ da enzima CPK-MB. Não houve variação significativa em ambas as enzimas, nos demais tempos.

\section{DISCUSSÃO}

A cardioplegia oxigenada tem sido referida como necessária para assegurar maior proteção ao miocárdio, durante a parada cardíaca, em cirurgia do coração ${ }^{2,}, 3$, 5, 6, 7, 9, 10 .

No presente estudo, procuramos analisar, comparativamente, 2 grupos de pacientes submetidos a cirurgia cardiaca, nos quais foi utilizada mesma solução cardioplégica $^{5}$, oxigenada no segundo grupo.

Os resultados demonstraram que ambos os grupos eram semelhantes quanto à idade, diagnóstico, grau funcional e tipo de cirurgia. O tempo de perfusão foi significativamente mais elevado no Grupo II $(P<0,05)$ e o tempo de parada cardiaca, embora maior no mesmo grupo, nảo foi estatisticamente significativo.

A análise dos dados referentes ao transoperatório evidenciou que a recuperação hemodinâmica foi normal em $100 \%$ dos casos do Grupo I e em $93 \%$ dos casos do Grupo II. O uso de drogas vaso-ativas foi maior no Grupo II, onde o cálcio e o nitroprussiato de sódio foram usados em $21 \%$ dos casos e a associação cálcio e dopamina ocorreu em $29 \%$ dos pacientes. No Grupo I, o cálcio foi usado em $25 \%$ dos casos e a dopamina, em $8 \%$.

No ECG, observou-se que o ritmo sinusal primário ocorreu em $33 \%$ dos pacientes do Grupo I e em $14 \%$ dos do Grupo II. A fibrilação ventricular seguida de ritmo sinusal ocorreu em $58 \%$ e $43 \%$ dos Grupos I e II, respectivamente. Observou-se, também, maior ocorrência de bradicardia sinusal no Grupo II (29\%), em relação ao 
COSTA, P. S.: PEREIRA, S. N.; MORAES, L. B.; MARQUES, R. S.; ALVAREZ, M. A. P.; DAUDT, C. A. S.; DEBONI, L M.; RASKOSKI, C.: SILVA, M. F. - A cardioplegia oxigenada na proteção miocárdica durante a cirurgia cardiaca: estudo clínico e enzimático. Rev. Bras. Cir. Cardiovasc., 3(3): 210-215, 1988.

Grupo I ( $8 \%$ ). A repolarização foi normal em $83 \%$ dos pacientes do Grupo I e em $93 \%$ dos do Grupo II. A desfibrilação elétrica foi necessária em $58 \%$ dos pacientes do Grupo I e em $92 \%$ do Grupo II (1 choque em $71 \%$ e 2 em $21 \%$ dos casos).

Estes achados se justificam, já que a perfusāo e a parada cardiaca foram mais prolongadas no Grupo II, levando à reinfusão da solução cardioplégica e, conseqüentemente, à maior incidência de bradicardia, logo após a parada cardiaca.

A análise dos dados de freqüência cardiaca, pressāo arterial média e pressão venosa central no pré e no pós-operatorio imediato, mostrou dados semelhantes, nos dois grupos, sem diferença significativa, em nenhum dos tempos.

O comportamento das enzimas TGO e CPK-MB demonstrou diferença significativa, com maior elevação no Grupo I, na amostra de pós-operatório imediato $(\mathrm{P}<$
$0,01)$ e da CPK-MB na de 6 horas de pós-operatório $(P<0,05)$. Estes achados sugerem que possa ter ocorrido lesão celular mais acentuada neste grupo do que no Grupo II.

Estes resultados estāo de acordo com os de TABAYASHI et alii ${ }^{10}$ e DE WITT et alii ${ }^{1}$, que consideram fundamental o papel da oxigenação da solução cardioplégica para a melhor proteção do miocárdio, em cirurgia cardiaca.

Em conclusāo, a maioria dos achados evidenciou um quadro muito semelhante entre ambos os grupos, com discreta vantagem em termos de recuperação transoperatória para o Grupo I. No pós-operatório, a variação das enzimas evidenciou maior elevação no Grupo I, que foi significativa, sugerindo maior lesão celular. Estes dados indicam que, provavelmente, o uso de cardioplegia oxigenada determine maior proteção ao miocárdio, em cirurgia cardiaca.

COSTA, P. S.; PEREIRA, S. N.; MORAES, L. B.; MARQUES, R. S.; ALVAREZ, M. A. P.; DAUDT, C. A. S.; DEBONI, L. M.; RASKOSKI, C.; SILVA, M. F. - Oxygenated cardioplegia in myocardial protection during cardiac surgery: a clinical and enzymatic study. Rev. Bras. Cir. Cardiovasc., 3(3):210-215. 1988.

ABSTRACT: Cardioplegia has been recognized as a very important factor in myocardial protection. Today we know that even the arrested heart at $15^{\circ} \mathrm{C}$ wastes oxygen. "In vitro" it was already shown that release of oxygen was higher in cristalloid than in blood solution. In this study we analized the hemodinamic, electrocardiographic and enzimatic variations in 26 patients, divided into two groups in whom cardioplegia with the Gomes solution was used. Group I: 12 patients (control group - non oxygenated solution). Grupo II: 14 patients (oxygenated solution). The evaluation of the patients included hemodynamic recovery after arrest, the use of vasoactive drugs, cardiac rhythm and aspect of the ECG, heart rate (HR), mean arterial pressure (MAP), central venous pressure (CVP) and enzymes SGOT and CPK-MB in the following times: pre and postoperatory period and after $6,12,24,48$ and 72 hours of postoperatory period. Hemodynamic recovery was similar in both groups, the use of vasoactive drugs was greater in Group II. In the ECG was seen more sinus bradycardia in Group II that aiso needed more electric defibrilation. Heart rate, mean arterial prossure and central venous pressure showed no significant difference between the two groups in the postoperative period. The enzymes SGOT and CPK were higher in Group I than in Group II. In conclusion, data suggested that both solutions had similar results in the hemodynamic recovery end vital parameters. An higher incidence of bradycardia was seen in Group II, probably related to the longer cardiac arrest, with more infusions of the cardioplegic solution. The variation of the enzimes suggested that oxygenated cardioplegia was more effective than the non-oxygenated solution to assure the preservation of the myocardium.

DESCRIPTORS: myocardial protection, cardioplegia

\section{REFERÊNCIAS BIBLIOGRÁFICAS}

1 DE WITT, L.; COETZEE, A.; KOTZ, J. \& LOSCHNER, A. - Oxigen requirements of the isolated rat heart during hypothermic cardioplegia: effect of oxigenation on metabolic and functional recovery after five hours of arrest. J. Thorac. Cardiovasc. Surg., 95:310-320, 1988.
2 DigeRnESS, S. B.; VANINI, V.; WIDEMAN, F. E. - "In vitro" comparison of oxigen availability from asanguineous and sanguineous cardioplegia media. Circulation, 64 (Supl. 2): 80-83, 1981.

3 FOLLETTE, D. M.; MULDER, D. G.; MALONEY Jr., J. V.: BUCKBERG, G. D. - Advantages of blood cardio. 
COSTA, P. S.; PEREIRA, S. N.; MORAES, L. B.; MARQUES, R. S.; ALVAREZ, M. A. P.; DAUDT, C. A. S.; DEBONI, L M.; RASKOSKI C. : SILVA, M. F. - A cardioplegia oxigenada na proteção miocárdica durante a cirurgia cardíaca: estudo clínico e enzimático Rev. Bras. Cir. Cardiovasc., 3(3): 210-215, 1988.

plegia over continuous coronary perfusion or intermitent ischemia: experimental and clinical study. J. Thorac. Cardiovasc. Surg., 76: 604-617, 1978.

4 GOMES, O.M. - Importância da oxigenação de soluçôes cardioplégicas para proteção miocárdica em cirurgia cardiaca. 11: Congresso Nacional de Cirurgia Cardíaca. Curitiba, 1983. (Resumo).

5 GOMES, O. M.; BARROS-MORAES, N. L. T.; FIORELLI, A. I.; BRUM, J. M. G.; RIBEIRO, M. P.; CALONGE, H. C. F.; ARMELIN, E.; BITTENCOURT, D.; ZERBINI, E. J. - Parada cardiaca anóxica: três horas de proteção. Nova solução cardioplégica. Arq. Bras. Cardiol., 36 (Supl. 1): 8-12, 1981.

6 KANTER, K. R.; JAFFIL, J. H.; GARLICHMAN, R. J.; FLATHERLY, J. T.; GOTT, V. L.; GARDNER, T. J. - Superiority of perfluorocarbon cardioplegia over blood or cristalloid cardioplegia. Circulation, 64 (Supl. 2): 75-79, 1981.

7 LEDINGHAM, S. J. M.; BAIMBRIDGE, M. V.; HEARSE, D. J. - The St. Thomas Hospital cardioplegic solution: a comparison of the efficacy of two formulations. J. Thorac. Cardiovasc. Surg., 93(2): 240-246, 1987.
8 MURAD, H.; QUEIROZ, A. D. R.; MATTOS, S. C.; PIEDADE, M. M.; ALBUQUERQUE, P. C. C.; MURAD, L. H. F.; PEREIRA, G. C.; BASTOS, E. S.; JAZBIK, A. P. - Proteção miocárdica por cardioplegia sangüinea durante circulação extracorpórea: avaliação ultra-estrutural miocárdica no cão. Arq. Bras. Cardiol., 41(4): 231-239, 1983

9 NELSON, R. I.; GOLSTEIN, S. M.; MCDONNEL, D. H. MALONEY Jr. J. V.; BUCKBERG, G. D. - Studies of the effect of hypothermia on miocardial regional blood flow and metabolism during cardiopulmonary bypass: $V$. Profound topical hypothermia during ischemia in arrested hearts. J. Thorac. Cardiovasc. Surg. 73(2): 201-207, 1977.

10 TABAYASHI, K.; MCKEOWN, P. P.; MIYAMOTO, M. LUEDTKE, A. E.; THOMAS, R.; ALLEN, M. D.; MISBACH, G. A.; IVEY, T. D. - Ischemic myocardial protection: comparison of nonoxigenated crystalloid, oxigenated crystalloid, and oxigenated fluorocarbon cardioplegic solutions. J. Thorac. Cardiovasc. Surg., 95(2): 239-246, 1988. 